\title{
Supporting Information for On the mechanism of negative differential resistance in ferrocenylundecanethiol self-assembled monolayers
}

\author{
Jin $\mathrm{He}^{1}$, and Stuart M. Lindsay ${ }^{1,2,3^{*}}$ \\ ${ }^{I}$ Department of Physics and Astronomy, ${ }^{2}$ Department of Chemistry and Biochemistry \\ and ${ }^{3}$ Biodesign Institute, Arizona State University, Tempe, AZ 85287, USA.
}

RECEIVED DATE (automatically inserted by publisher);Stuart.Lindsay@asu.edu

\section{Preparation and characterization of SAMs}

Ferrocenylundecanethiol was purchased from Dojindo Co. (Atlanta, GA) and used as-received. It was dissolved to a final concentration of ca. $0.8 \mathrm{mM}$ in freshly distilled and Ar bubbled Tetrahydrofuran (THF) and deposited onto a freshly hydrogenflame-annealed $\mathrm{Au}(111)$ substrate $^{1}$ for $12 \mathrm{~h}$ at room temperature. Cyclic voltammograms showed the reversible ferrocene oxidation, while the integrated currents indicated monolayer formation. SAMs were rinsed copiously with THF, dried in an argon stream and then placed in the liquid-cell of the STM under mesitylene, maintained under nitrogen as described below. The STM tips were formed by cutting a $0.25 \mathrm{~mm}$-diameter Au (Alfa Aesar, 99.999\%) wire. They were then immersed in Piranha Solution (3:1 $\mathrm{H}_{2} \mathrm{SO}_{4}: \mathrm{H}_{2} \mathrm{O}_{2}(30 \%)$ by volume) for $30 \mathrm{~s}$, rinsed with DI water and dried in Ar stream prior to use. Caution! Piranha solution is a very strong oxidant and is extremely dangerous to work with; gloves, goggles, and a face shield should be worn.

\section{Data correction for charging}

Raw scans (Fig. S1a) were distorted significantly by charging current. The charging current was measured by withdrawing the tip from the surface to eliminate tunneling current (Fig. S1b). The resulting iv curve shows a steady-state charging current proportional to $\mathrm{dV} / \mathrm{dt}$ where the magnitude of the current yields a capacitance of $0.1 \mathrm{pF}$. The turn-around points are well fitted by an exponential with a time constant of $1 \mathrm{~ms}$ (corresponding to an interfacial resistance of $1 \mathrm{G} \Omega$ ). Subtraction of this current yields the corrected scan shown in Fig. S1c. This correction fails when there is significant tunneling current in addition to the leakage, giving rise to the appearance of a small spurious peak near the turn around points.

\section{Removal of oxygen}

The local oxygen concentration was determined using cycling voltammetry of iron-porphyrin monolayers. The iron redox peak is extremely sensitive to $\mathrm{O}_{2}$ as manifested by a loss of charge under the reduction peak on the return sweep. Ultra high pure $\mathrm{N}_{2}$ (99.999\%) was slowly passed through an Oxiclear gas purifier (Labclear Inc., CA.) and then passed through the environmental chamber of the PicoSPM. We also inserted a small teflon tube directly into the liquid in the sample cell, and bubbled deoxygenated Ar through the mesitylene. The sample was de-gassed for a period of $3 \mathrm{~h}$ prior to measurement. De-gassed mesitylene was added through the small Teflon tube every hour to compensate for the evaporation of mesitylene. $50 \mathrm{~mL}$ pyrogallol alkaline solution (2.7g $\left.\mathrm{C}_{6} \mathrm{H}_{6} \mathrm{O}_{3}, 38 \mathrm{~g} \mathrm{KOH}\right)$ was used as an additional oxygen absorber. It was activated in a glovebox and then sealed into the environmental chamber using an interlock system built for this microscope (Molecular Imaging, Tempe, AZ). Colorless pyrogallol alkaline solution became light brown over the course of an experiment, turning dark red when exposed to air after the experiment.

(1) DeRose, J. A.; Thundat, T.; Nagahara, L. A.; Lindsay, S. M. Surf. Sci. 1991, 256 102-108.
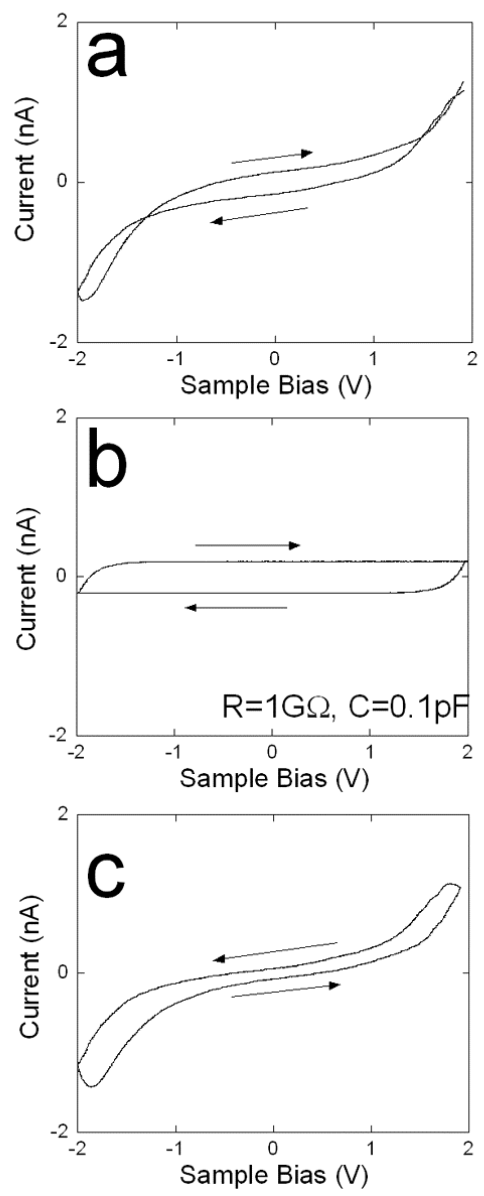

Figure S1: Correction for charging. (a) shows raw data. (b) shows the charging current measured with the probe withdrawn from the surface. It is well-fitted with $\mathrm{R}=1 \mathrm{G} \Omega$ and $\mathrm{C}=0.1 \mathrm{pF}$. (c) shows the data in panel (a) after subtraction of the current shown in (b). The small peaks near the turn around points are an artifact of this subtraction. 

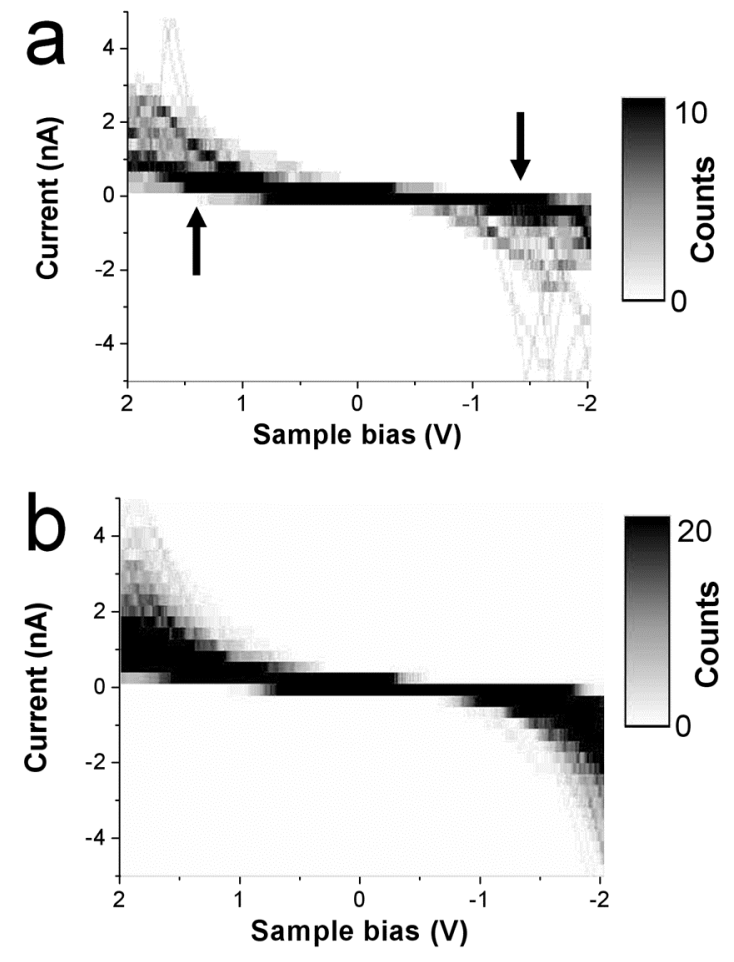

Figure S2: Statistical summary of the downward scans (c.f. Figs. 1d, 2b) in (a) ambient conditions and (b) oxygen-free conditions. The same 2D histogram display is used here as in Figs $1 \mathrm{~d}$ and $2 \mathrm{~b}$. Arrows mark the NDR peaks at ca $\pm 1.5 \mathrm{~V}$ 